\title{
Prozessorientiertes Marketing
}

Marketing wird komplexer: mehr Produkte und Services, mehr und verschiedene Kunden, mehr Länder, mehr einbezogene Abteilungen. Parallel steigen mögliche Ansätze im Marketing ins Unermessliche.

Um mit Vielfalt sinnvoll umgehen zu können, braucht es substanzielle Bezüge. Ein erster Bezug besteht in einer kraftvollen (Marketing-)Strategie. Der nächste Bezug wäre eine klare Marketing-Logik, die mindestens die Haupt- und Nebeninstrumente des Marketing bestimmt. Schließlich sollen Kernprozesse die Aktivitäten von vielen Beteiligten im Unternehmen und den Kunden verbinden. Bereits die frühen Diskussionen zur Prozessorientierung prägten Hinweise zum Ende von Abteilungs-Silos oder zu sinnvollen Schnittstellen. Gut geführte Prozesse berücksichtigen die Dynamik, sie konzentrieren sich auf wichtige Aufgaben, sie orientieren sich an Ergebnissen und damit bewegen sich Unternehmen schneller und wirtschaftlicher.

In der Produktion oder Informatik werden Prozesse schon länger definiert. Auch im Marketing sind besonders interne Prozesse gut geregelt, etwa der Auftragsablauf. Anspruchsvoll und gleichzeitig besonders ergiebig sind jedoch Prozesse, die nicht nur vom Unternehmen, sondern auch vom Kunden geprägt werden. Etwa: Wie führen wir die Kunden zum Kauf? Wie erreichen wir rasch den Break Even für neue Produkte? Lässt sich ein Auslandsmarkt schneller und besser erschließen? Manche dieser Prozesse werden bisher zu wenig beachtet. Andere wurden einfach dezentral entwickelt, die Standardisierung vereinfacht und das Vorgehen professionalisiert. So lassen sich beispielsweise Evaluationen für neue Märkte systematisieren und mit Informatik unterstützen. Zudem gilt es, eingespielte Prozesse zu prüfen. Beispielsweise sind aktuelle Budgetierungen häufig zu aufwendig und für die Führung kaum mehr ergiebig.

Prozessorientiertes Marketing ist ein breites und wichtiges Thema. Dabei kann dieses Heft nur wenige Akzente setzen. Stichworte dazu sind: kundenprozessorientiertes Marketing, Prozessorientierung im Online-Geschäft, Umgang mit Kundenströmen im Destinationsmanagement, Vertriebsprozesse

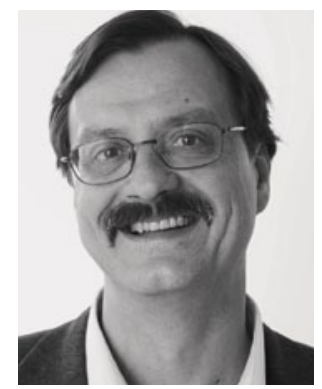

Prof. Dr. Christian Belz

Ordinarius für Marketing und Direktor

am Institut für Marketing,

Mitbegründer und -herausgeber der

Marketing Review St. Gallen

christian.belz@unisg.ch

nach Geschäftstypen, Prozess des Value Sellings. Wir hoffen, dass diese spezifischen Beiträge den Leser zu eigenen Neuerungen anregen. Noch besser wäre der Effekt, dass sich Verantwortliche in Marketing und Vertrieb überlegen:

- Welche Herausforderungen können wir mit einem Prozessansatz besser bewältigen?

- Welche Kernprozesse müssen wir bestimmen? Wie laufen aktuelle Prozesse und welche Ziele sind zu erreichen?

- Welche Menschen und Einheiten müssen für diese Prozesse im Unternehmen und beim Kunden zusammenwirken?

- Wie strukturieren wir die ausgewählten Kernprozesse? Wie definieren wir die Aufgaben? Wie bestimmen wir das $\mathrm{Zu}$ sammenspiel?

Kurz: Wir sind überzeugt, dass sich diese Betrachtung für Unternehmen und Manager lohnt. Wir freuen uns über Ihre Hinweise und eigene Beispiele dazu.

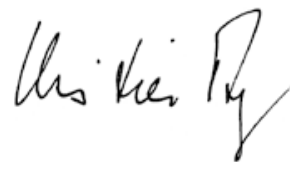

